\title{
The role of multicomponent surface diffusion in growth and doping of silicon nanowires
}

\author{
A. Efremov ${ }^{1}$, A. Klimovskaya ${ }^{1}$, D. Hourlier ${ }^{2}$ \\ ${ }^{1} V$. Lashkaryov Institute of Semiconductor Physics, NAS of Ukraine \\ 45, prospect Nauky, 03028 Kyiv, Ukraine \\ ${ }^{2}$ Institut d'Electronique, de Microélectronique et de Nanotechnologies, ISEN, \\ UMR-CNRS 8520, F-59652 Villeneuve d'Ascq, France
}

\begin{abstract}
The metal-catalyzed chemical vapor deposition on silicon substrates remains one of the most promising technologies for growing the silicon nanowires up to now. The process involves a wide variety of elementary events (adsorption, desorption, and multicomponent atomic transport with strongly different local mobility, etc.) that take place on the same surface sites and proceed on isolated nano-scaled part of the surface belonging to different individual catalyst particle. In this work, the competition for unoccupied sites during atomic transport under growth doping and percolation-related phenomena on confined parts of surface was treated by the Monte-Carlo simulations. Atomistic simulations were compared with numerical kinetic modeling. Arising nonlinear effects that finally lead to specific modes of the nanoobject growth, shaping, and doping were analyzed. By combining different kinds of simulations and experimental results, the proposed strategy provides a better control at atomic scale of nanowire growth. Both atomistic and kinetic considerations supplementing each other reveal the importance of surface transport and the role of surface immobile contaminations in the nanowire growth.
\end{abstract}

Keywords: nanowire growth, doping, surface transport, Monte-Carlo simulations, kinetic modeling.

Manuscript received 14.11.06; accepted for publication 26.03.07; published online 01.06.07.

\section{Introduction}

Metal-catalyst mediated, quasi-one-dimensional growth of arrays of nanowires (NWs) by chemical vapor deposition (CVD) allows to create a wide variety of forms and shapes of nanowires [1], varying from straight, nearly circular cylinders to prisms, telescopelike objects, cones, bead-like free-standing filaments, springs [2, 3] or even single spheres [4]. In some cases, nanostructures with "core-shell" structure $[5,6]$ were observed. As a rule, all of these various objects are formed spontaneously without any special efforts, due to some unaccounted factors. Therefore, theoretically the desirable shape of the nanowires may be achieved relying upon only internal mechanisms of selforganization without strong external force measures. However, there is no clear understanding up to now what factors should be involved to achieve this goal together with the good uniformity of size and length and to realize all the very promising potentialities of this technique.
The increasing interest to nanowire doping during growth, for example with boron or phosphorous, gives rise to another important frame of problems. They are the co-adsorption of different kinds of molecules and the multicomponent transport of different sorts of atoms toward catalyst/silicon interface or nanowire sidewall. Multiple mutual influences, competition for unoccupied sites both during adsorption and surface transport, percolation related phenomena promise a number of non-linear effects, which may result in unexpected consequences both in shaping and doping. The simplest effect of such kind is the selective adsorption of molecules from gas phase onto some vacancy-enriched regions of the surface, which are naturally formed near diffusion sinks.

In this paper, we discuss some key mechanisms responsible for nanowire shaping and doping in CVD nanowire growth, when silane or other hydrogencontaining active gas (disilane, borane, or phosphine) is used. As a result, after chemosorption of these molecules at least three groups of adsorbed species are formed on 
the catalyst surface: (1) low-mobile complexes $\left(\equiv \mathrm{Si}-\mathrm{H}_{\mathrm{n}}\right.$, and possibly dopant-containing species), (2) adatoms with a mean but different local mobility (Si, B, P), and (3) hydrogen adatoms with a high local mobility, which are also able to escape from the surface by associative desorption. All of these species can occupy the same set of adsorption and diffusion sites, impeding each other just in surface transport, and resulting in different feedbacks in the growth process. Respective fluxes of substance, determining the growth of NW are schematically shown in Fig. 1.

We restricted our consideration by the processes upon the surface of catalyst particle. The detail analysis of exactly these processes should give us a lead to reproducible synthesis of NW with unexpected forms [2-6]. In comparison with the other parts of a nanoobject here the most intensive active gas decomposition takes place, and the highest concentration of mobile silicon (or impurity) adatoms is sustained. The control over further redirection of these atoms to the interface or to the sidewall seems to be looked for a way to the shape and doping quality control.

\section{Modeling}

The growth process of the NW as a whole and some of its stages may be treated in the framework of a kinetic approach by numerical solution of respective balance equations [1], while adequate consideration of the surface transport across catalyst needs another way of looking. Here, concentrations of several different species may be high enough, and their mobilities differ each other arbitrary. In this special case, the surface transport could not be described as ordinary diffusion and belongs to the so-called "strange processes". It may be described kinetically in the framework of fractional dynamics or strange kinetics [7]. In particular, the Einstein relation transforms in [7]:

$$
\left\langle R^{2}\right\rangle=4 D_{\text {eff }} t^{\mu}
$$

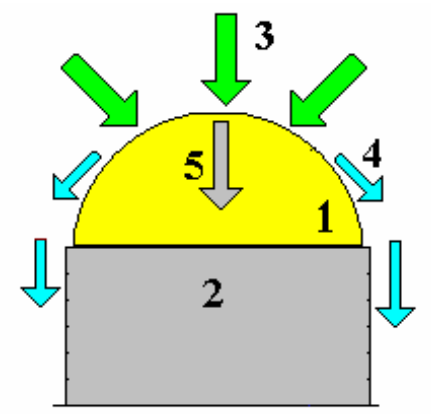

Fig. 1. Distribution of molecular and atomic fluxes adopted in the kinetic model for nanowire growth. 1 - a metal catalyst particle or droplet, 2 - a nanowire body, 3 - molecular adsorption from the gas phase, 4 - the surface transport of silicon adatoms to the nanowire sidewall, 5 - the bulk transport of silicon atoms to the metal/silicon interface.
Here $\left\langle R^{2}\right\rangle$ is the mean square displacement during time $t, D_{\text {eff }}$ is an effective transport coefficient, and in a general case $\mu \neq 1$. For arbitrary complex multicomponent systems, fractional dynamics encounters with insuperable difficulties. However, the peculiarities mentioned above, including evolution of detail spatial distribution of adatoms on the surface of catalyst, may be directly studied by the MC simulations. We advisedly restrict the application of the MC simulations by this part of growing nanoobject and only of this key stage of the process, instead of using the atomistic modeling for the growth process as a whole. We will apply the MC simulations here in order (i) to estimate the values of some physical parameters (transport coefficients dependences) with their subsequent use in kinetic modeling, and (ii) to reproduce some key fragment of real physical experiment. Besides, we will demonstrate some advantages of "multilevel modeling" that combines the atomistic and macroscopic approaches within the same model and allows to avoid some difficulties of strange kinetics formalism.

In the model of catalyst particle surface transport, the following elementary events are selected:

(i) Adsorption from the gas phase onto a single random unoccupied site. Generally speaking, the real act of adsorption may need a group (two or more) of unoccupied neighboring centers, for example for accommodation of hydrogen atoms, just after silane decomposition. The detail numerical analysis has shown [8] that different scenarios of silane adsorption onto silicon surface result in practically the same growth kinetics. For this reason, we content with this simplest model for adsorption. The input flux is measured in the number of impinging atoms per one lattice site per one MC-time step $\tau$.

(ii) Randomly directed atomic jump with the length $\lambda$ (the distance between the nearest sites) of the random atom to the nearest unoccupied site.

(iii) In the general case, the irreversible going away of the random atom from the surface to the bulk of catalyst.

The MC-simulations including the processes above were carried out in a two-dimensional $100 \times 100$ lattice, which corresponds to real NW diameter about $20 \mathrm{~nm}$.

We made four types of numerical experiments, complementing each other, namely:

(i) Migration of the probe particles across the surface with different coverage up to the first touch of the boundary of the region, with the radius $R$ and averaging the respective time of the path $\langle t\rangle$ [9]. Other atoms make atomic jumps with the local mobility $D_{\text {loc }}=\frac{1}{4} \lambda^{2} / \tau$ being a background for movement of a studying probe particle with the same or another local mobility. Here adsorption is not considered. This simulation allowed us to estimate the effective transport coefficient $D_{\text {eff }}$ and exponent $\mu$, which may be attributed to this surface (Fig. 2a). 

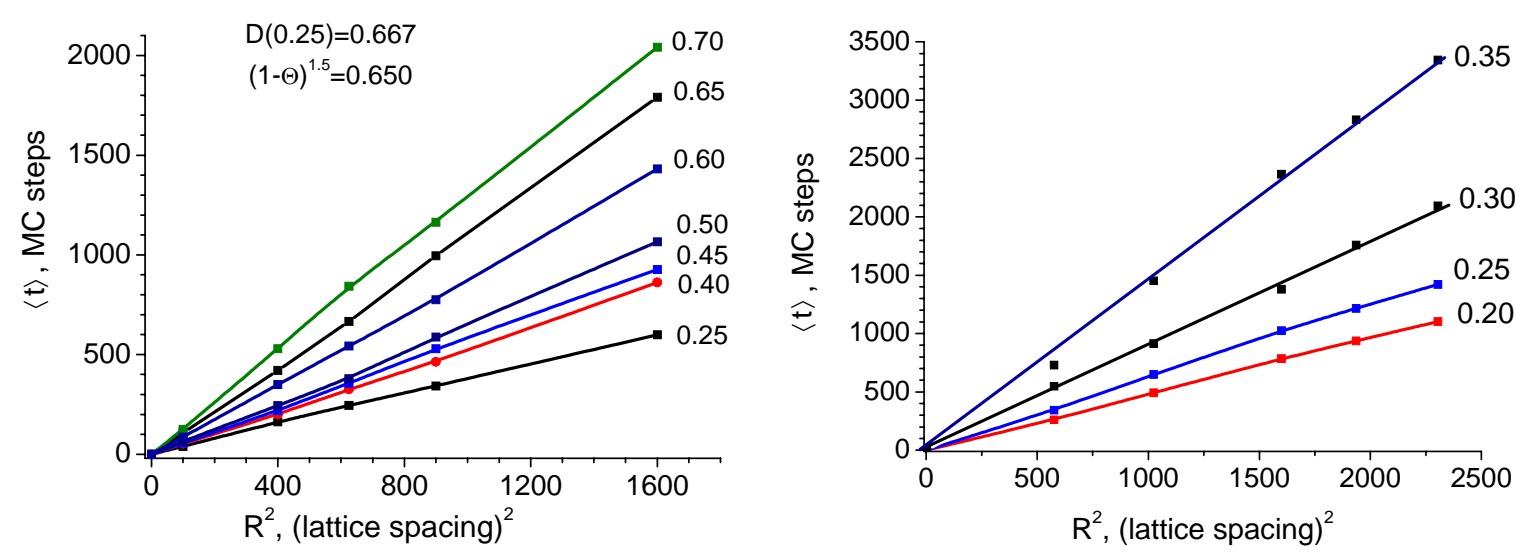

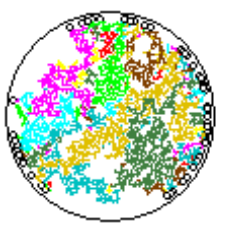

0.38



0.42

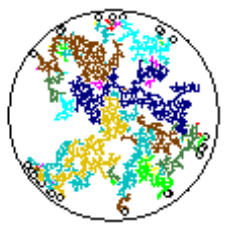

0.41

$b$
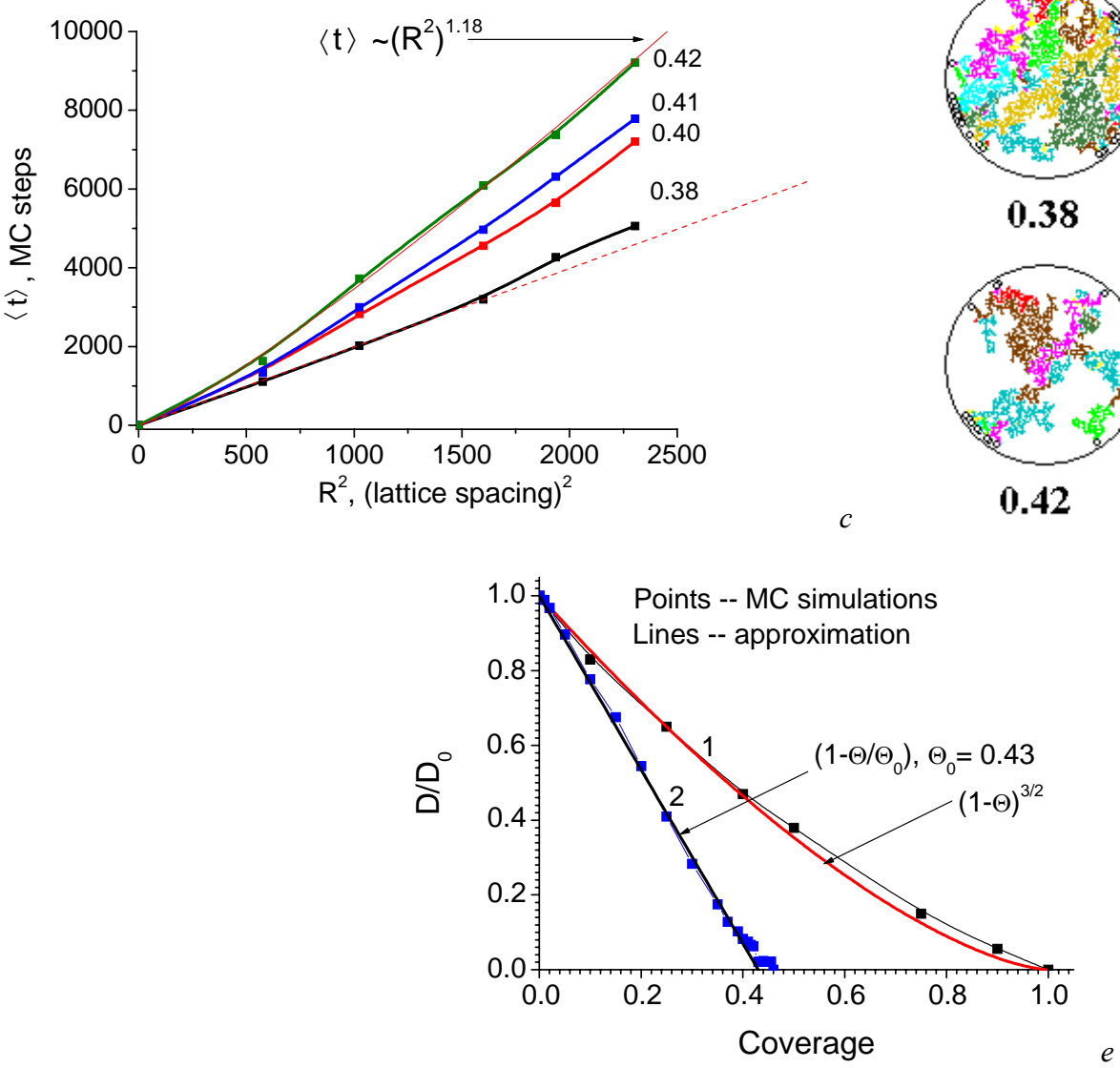

Fig. 2. Results of MC-simulations as to the surface transport on catalyst particle surface.

a - Relationship between $\langle t\rangle$ and $R^{2}$ for $0.25 \leq \Theta \leq 0.70$ when the random-walk of a probe particle is carried out on the surface covered with one sort of random-walking atoms. The slope of the lines is equal to $\left(4 D_{\text {eff }}\right)^{-1}$.

b - Relationship between $\langle t\rangle$ and $R^{2}$ for mobile random-walking probe particle at $\Theta_{\text {slow }} \leq 0.35$ when the surface is covered with slow-moving impurities as a background. The slope of the lines is equal to $\left(4 D_{\text {eff }}\right)^{-1}$ as before.

c - Relationship between $\langle t\rangle$ and $R^{2}$ for mobile random-walking probe particle on the surface covered with slow-moving impurities at $0.35<\Theta_{\text {slow }}<0.43$. Effective diffusivity may be obtained from (1).

$\mathrm{d}$ - Spatial distribution of allowed trajectories for the mobile random-walking particles on the surface covered with slowmoving impurities at $\Theta_{\text {slow }}=0.38,0.41,0.42$, and 0.43 , respectively.

e - Dependence of the effective diffusivity on the surface coverage derived from curves in (a)-(c) and its analytical approximation. Conditions: (1) probe particle migration on the lattice covered with mobile atoms under periodical boundary conditions; (2) migration of probe mobile particle across a surface primarily covered by the nearly immobile species. 
(ii) Classical MC experiment $[9,10]$ when random trajectories of all moving atoms are monitored, and averaged values $\left\langle R^{2}\right\rangle$ or $\langle t\rangle$ are calculated. These results were compared with the previous case.

(iii) The measurements of the non-stationary atom escaping kinetics of one (mobile) component $\Theta_{1}=\left.F_{1}\left(\Theta_{1}, \Theta_{2}, \ldots, t\right)\right|_{J_{\text {inp }}=0}$ at zero input flux of molecules from gas phase and nonzero initial coverages (Fig. 3). In this case, the circle region on the surface was randomly covered with two sorts of adatoms $\Theta_{1}, \Theta_{2}$ with different local mobility and then their migration up to leaving the considered region was monitored.

(iv) The calculation of stationary relationship $J_{\text {out }}^{(0)}=F\left(J_{\text {inp }}, D_{\text {loc }}\right)$ between input flux of adatoms (which appear after molecule dissociative adsorption), stationary output surface flux beyond the bounds of a catalyst particle (Fig. 1, arrow \#4); and between $J_{\text {inp }}$ and respective steady-state coverage, which is established at that: $\Theta_{1}^{(0)}=\left.\varphi\left(J_{\text {inp }}, D_{\text {loc }}\right)\right|_{t \rightarrow \infty}$ (Fig. 4a, b). The first two approaches allow us to derive transport coefficients and use them in kinetic equations. The second two simulations correspond in higher degree to a real experiment and allow us to compare atomistic simulations and kinetic modeling.

\section{Results and discussion}

3.1. Effective transport coefficients and their use in a kinetic model of the $\mathrm{NW}$ growth

Experiments with a probe particle in the lattice covered with atoms having resemble mobilities show that the Einstein relation $R^{2} \propto D_{\text {eff }} t(\mu \approx 1.00 \pm 0.01)$ is valid within the wide range of the coverage. The respective

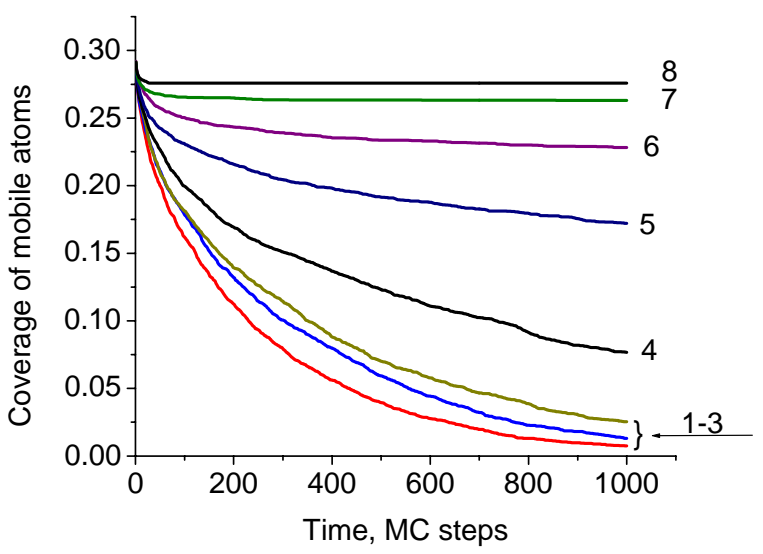

Fig. 3. Dependence of the spread kinetics (smoothed by median filter) of surface mobile atoms on the surface preliminary covered with immobile species according to MC. Initial coverage $\Theta_{\text {mob }}=0.3$ and $\Theta_{\text {slow }}=0.0 ; 0.10$; 0.20 ; 0.30 ; 0.40 ; 0.50 ; 0.60 and 0.69 (1-8).

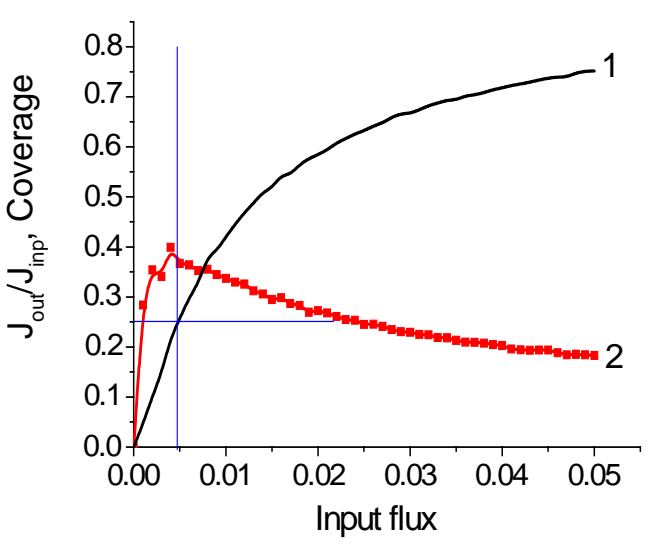

a)

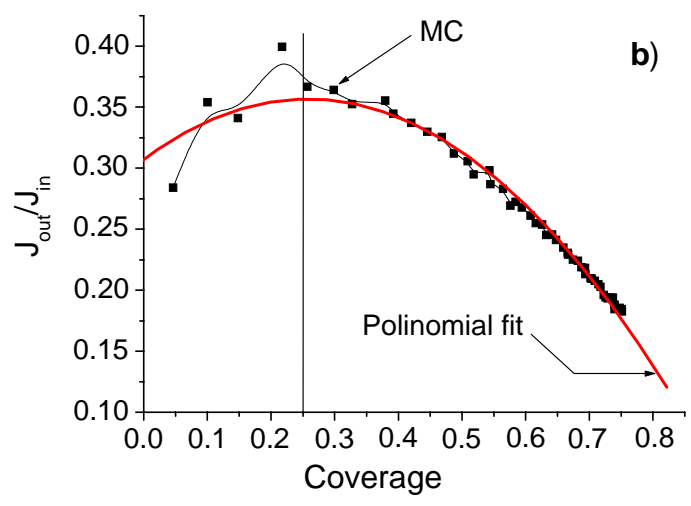

Fig. 4. Surface coverage (1) and normalized output flux ( $J_{\text {out }} / J_{\text {inp }}$ ) (2) established due to surface transport as a function of the input flux (a) and normalized output flux as a function of the corresponding steady-state surface coverage (b).

dependences for $\langle t\rangle=f\left(R^{2}\right)$ at $0.25 \leq \Theta \leq 0.70$ are shown in Fig. 2a. We did not observe any sufficient deviation from the proportionality $\langle t\rangle \propto R^{2}$ even in the vicinity of the percolation threshold. The effective transport coefficient $D_{\text {eff }}$ is the monotonously decreased function of $\Theta$ presented in Fig. 2e (curve 1). This behavior that has a strong resemblance to the ordinary Gaussian diffusion is well known for transport processes on percolating fractal sets [7]. In spite of this similarity with simple diffusion, the commonly used diffusion equation $\frac{\partial \Theta}{\partial t}=\nabla(D(\Theta) \nabla \Theta)$ is not valid in the vicinity of percolation threshold [7] and true time evolution of detailed surface distribution of migrating atoms may be considered correctly within the "fractional dynamics" approach. Nevertheless, a crude kinetic description (without fine spatial details) is possible, for all the $\Theta$-range, because $\langle t\rangle$ corresponds to an average residence time of a migrating atom within a hemispherical surface region of the radius $R$, till the moment when the atom leaves the surface of the catalyst particle. 
Let us assume atomic fluxes distribution as shown in Fig. 1.

Applying some analytical approximation of the obtained dependence for effective diffusivity (e.g. $D_{\text {eff }}=D_{0}(1-\Theta)^{n}$, Fig. 2e), one may obtain the simple balance equation for an average adatom concentration within some semispherical region of the radius $R$ as shown in Fig. 1. The average time $\langle t\rangle$ depends on $R^{2}$ and $D_{\text {eff }}$ exactly as a quasi-steady-state diffusion time does [11]. This allows us to apply the following "crude" (without fine spatial details) kinetic model for the average surface concentration:

$\sigma \frac{d \Theta}{d t}=J_{\text {inp }}(1-\Theta)-\sigma \Theta(\beta+1 /\langle t\rangle)$,

$\frac{d h}{d t}=2 \beta \sigma \Theta \Omega$.

Where $\sigma$ is the surface site density available both for diffusion and adsorption, $\beta$ is the bulk transport rate constant for surface atoms through the body of a catalyst particle (Fig. 1, arrow \#5). The input flux $J_{\text {inp }}$ corresponds to a number silicon adatoms really appearing on the empty ( $\Theta=0$ ) surface of catalyst per unit time and per unit of area due to dissociative adsorption of pattering molecules (Fig. 1, arrow \#3). In the single-reaction approximation [1, 12], when chemosorption is accompanied by the only overcome one potential barrier $E_{a}$, this flux may be written as:

$J_{\text {inp }}=J_{\text {mol }} \cdot \exp \left(-\frac{E_{a}}{k_{\mathrm{B}} T}\right)$,

where $J_{\text {mol }}$ is the flux of pattering molecules, and $T$ is the temperature of a catalyst surface.

For $\beta$, this estimate may be approved

$\beta \approx \kappa \frac{D_{b}}{R \cdot \lambda_{b}}$

where $D_{b}$ is the bulk diffusivity of catalyst material, $\lambda_{b}$ is its lattice spacing in the bulk of catalyst and $\kappa$ is a multiplier of about $1 / 2$.

The surface transport is described by the reciprocal average residence time $\langle t\rangle^{-1}=\alpha D_{0} \cdot(1-\theta)^{n} / R^{2}$, where $\alpha=\frac{\mu_{0}^{2}}{(\pi / 2)^{2}} \approx 4.615$ is the shape coefficient for diffusion-time approximation in the case of the hemispherical region [11], and $\mu_{0}$ is the first root of the zero-order Bessel function. The second equation of the system describes the elongation growth rate of the nanowire due to atoms delivered to the catalyst/silicon interface by the bulk diffusion through catalyst. Here, $h$ is the nanowire height, and $\Omega$ is the atomic volume of silicon atoms in silicon, the multiplier 2 is the surface/interface areas ratio for the hemispherical catalyst particle. The steady-state $\left(\frac{d \Theta}{d t}=0\right)$ equation that corresponds to a constant elongation growth rate are the non-linear one with $J_{\text {inp }}$ as a parameter looks as follows:

$\frac{J_{\text {inp }}}{\sigma}=\kappa \frac{D_{b}}{R \cdot \lambda_{b}} \frac{\Theta}{(1-\Theta)}+\frac{\alpha D_{0}}{R^{2}} \cdot \Theta \cdot(1-\theta)^{n-1}$

or in a dimensionless form:

$\frac{J_{\text {inp }}}{J_{0}}=\left(1-E_{s b}^{r}\right) \frac{\Theta}{(1-\Theta)}+E_{s b}^{r} \cdot \Theta \cdot(1-\theta)^{n-1}$.

Where $E_{s b}^{r}=t_{b} /\left(t_{b}+t_{s}\right)$ corresponds to the share of a surface mass transfer channel in a total mass transfer, $t_{b}=\frac{R \cdot \lambda_{b}}{\kappa D_{b}}$ is the characteristic time of the bulk diffusion toward the interface, and $t_{s}=\frac{R^{2}}{\alpha D_{0}}$ is the respective characteristic time of the surface transfer toward the catalyst/sidewall boundary. The relative role of the surface transport may be characterized by the ratio $t_{b} / t_{s}=\frac{\alpha}{k} \frac{\lambda_{b}}{R} \frac{D_{0}}{D_{b}} \approx 0.1 \frac{D_{0}}{D_{b}} . J_{0}=\sigma / t_{\text {eff }}$ is a characteristic flux, where $t_{\mathrm{eff}}=t_{s} t_{b} /\left(t_{s}+t_{b}\right)$ is an effective residence time of an adatom on catalyst particle surface. One can see that the relative role of the surface transport increases with downsizing the catalyst particle.

The steady-state coverage $\Theta^{(0)}$ as a function of the normalized input flux $J_{\text {inp }} / J_{0}$ calculated numerically according to the model (4) at $n=1.5$ (see Fig. 2e, curve 1) is shown in Fig. 5.

One can see that the system behavior is rather trivial, while $E_{s b}^{r} \leq 0.983$. The latter corresponds to $t_{b} / t_{s} \leq 57.82$. This threshold value is realized, for example, at $R \leq 172 \lambda_{b}(R \leq 35 \mathrm{~nm})$ and $D_{0} / D_{b} \approx 10^{3}$. At any input flux, only one steady-state solution for coverage takes place in this case, while at $J_{\text {inp }} / J_{0} \geq 0.4$, a strongly non-linear $\Theta=f\left(J_{\text {inp }}\right)$ dependence is observed. At higher values of $t_{b} / t_{s}$ important quality changes occur. In this case, there is some narrow critical interval of the input flux $0.3 \leq J_{\text {inp }} / J_{0} \leq 0.4$ just near the percolation threshold $\left(\Theta_{C}=1 / 2\right)$, where the sharp step is observed on the $\Theta=f\left(J_{\text {inp }}\right)$ dependence. Inside this interval, the steady state equation has three solutions $x_{1}, x_{2}$ and $x_{3}$, corresponding to the given $J_{\text {inp }}$ (Fig. 5a). Any of these 

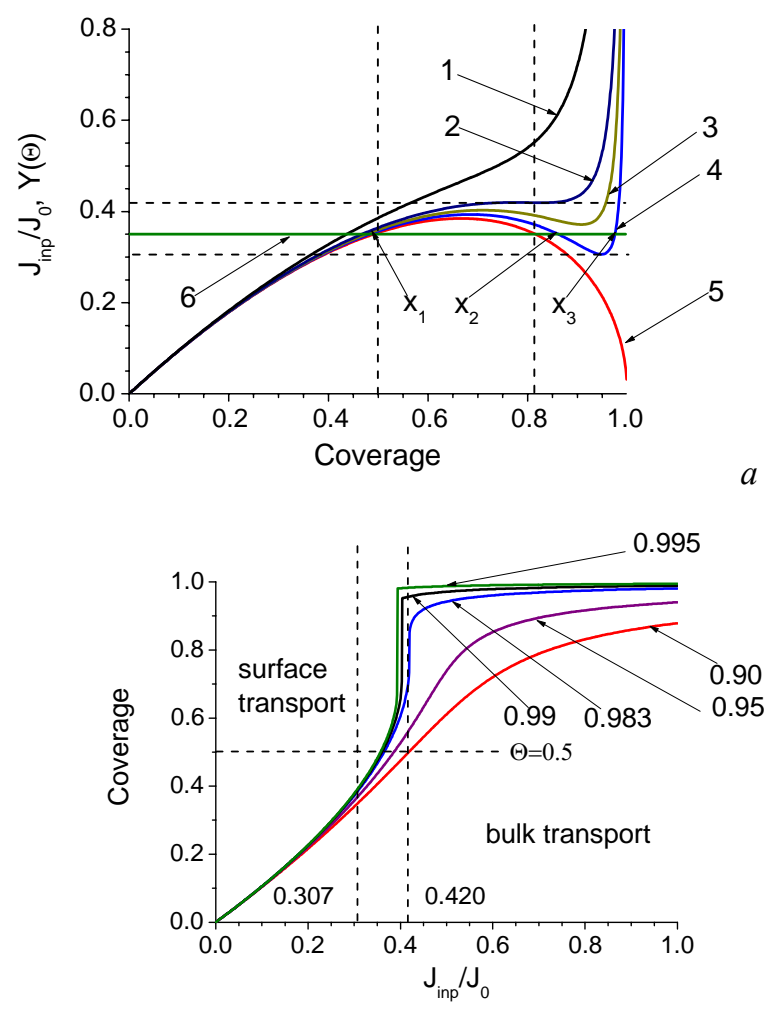

$b$

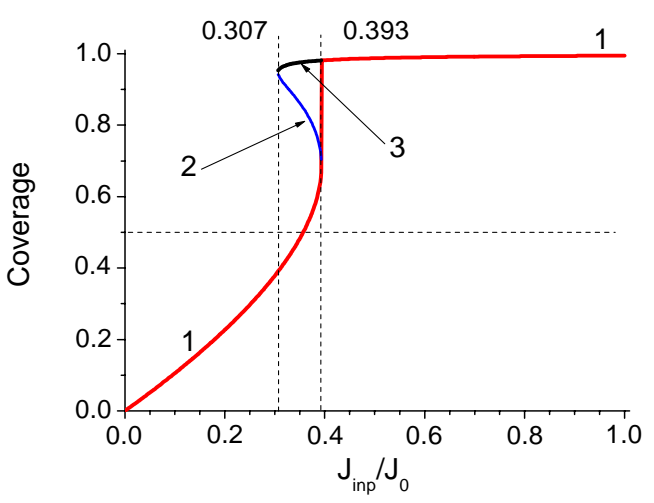

Fig. 5. Solutions of the steady-state Eq. (4). (a) Graphic illustration for solution at different values of $E_{s b}^{r}$. Curves 1-5 are $Y(\Theta)$ corresponding to the right-side of the equation, and 6 is $J_{\text {inp }} / J_{0}$ corresponding to the left one. Intersection points are respective steady-state solutions $x_{1}, x_{2}, x_{3}$ for the given input flux. Curves 1-5 conform to $E_{b s}^{r}=0.95,0.983,0.99$, 0.995, 1.00, respectively. (b) Relationship between resulting surface coverage and the normalized input flux obtained as the first root of Eq. (4) at different values of $E_{s b}^{r}$, indicated by numbers near curves. (c) Dependence of the surface coverage on $J_{\text {inp }} / J_{0}$ at $E_{s b}^{r}=0.99$, when all three solutions take place. 1,2 and 3 are $1^{\text {st }}, 2^{\text {nd }}$ and $3^{\text {rd }}$ solutions, respectively, at the given input flux. The region of negative slope and hysteresis are observed on the dependence. solutions may be realized depending on the initial conditions $\left.\Theta(t)\right|_{t=0}$ for Eq. (4). As may be shown, if $\left.\Theta(t)\right|_{t=0}=0$ the smallest steady-state coverage $\Theta^{(0)}=\left.\Theta(t)\right|_{t=\infty}=x_{1}\left(J_{\text {inp }} / J_{0}\right)$ corresponding to the first root is realized (Fig. 5b). If the initial condition is chosen so that $x_{1} \leq \Theta(0) \leq x_{2}$, or $\Theta(0) \geq x_{3}$, the second or third solution would be realized respectively in the steadystate. As follows from (4), the steady-state axial growth rate is proportional to $\Theta^{(0)}$, but the surface transport (and therefore, the delivery of adatoms to the sidewalls) is a rather complicated non-linear function of the coverage. Above the percolation threshold, the surface leakage of adatoms is partially blocked and looks like diffusion of vacancies. Even small flux fluctuations in the critical region should result in large amplitude variations in $\Theta$ and all the related parameters, including radial and axial growth rates. Above the percolation threshold, the system sharply turns to the state with a rather high coverage $(\Theta \approx 0.8 \ldots 0.9)$ and high elongation growth rate. Here, the surface transport proves to be suppressed, and efficiency of adsorption of molecules that is proportional to $(1-\Theta)$ decreases, too. Further increase of input flux results in insufficient increase of coverage by silicon adatoms. Therefore, the system demonstrates a trigger-like behavior as to transition through percolation threshold and some discrete spectrum of axial growth rates depending on initial conditions.

There are at least three important consequences for NW growth, which may be derived from this non-linear behavior:

(i) Catalyst particles (or liquid eutectic drops) may absorb silicon not only from the gas phase but from silicon substrate, too. The former process dominates during growth, but the latter may be important during different pre-growth treatments of the substrate covered with catalyst material film. Therefore, some spread in the initial conditions as to the surface coverage of different drops results in unexpected discrete spread in $\Theta^{(0)}$ and, hence, in axial growth rates under critical input flux and unfavorable combination of transport coefficients.

(ii) Small radius NWs easily demonstrate a nonlinear growth behavior, and a sharper step on the $\Theta=f\left(J_{\text {inp }}\right)$ dependence is observed, because $E_{s b}^{r} \rightarrow 1$ for them (Fig. 5a, curves 4,5). Besides, in this case the critical value of input flux may be rather small, but the difference between $x_{1}$ and $x_{2}$ increases sufficiently. Therefore, both long and very short NWs may be grown on the same substrate on neighbor catalyst drops even with the same radius. Only a very precise choice of the input flux and initial conditions (when only one steadystate solution takes place) may guarantee uniform axial growth in small-radius NW ensemble. 
(iii) The complete dependence for $\Theta=f\left(J_{\text {inp }}\right)$ uniting all three solutions is shown in Fig 5c. It demonstrates some hysteresis and the presence of some part with the negative slope. The latter create the necessary prerequisites for chemical, shape and growth auto-oscillations in such a system.

MC simulations, considering adsorption and diffusion inside restricted region of external surface of catalyst particle with the irreversible sink toward NW sidewall, have demonstrated both similarities and important differences from the kinetic modeling (2)-(4). Experiments for flux vs. flux dependences have shown that under high input fluxes the surface transport, while being strongly suppressed, does not disappear completely in the finite dimension systems.

First of all, adatoms distribute inhomogeneously along the surface $\Theta=\Theta(r)$. Due to boundary sink of atoms toward the NW sidewall, some narrow vacancyenriched strip at the periphery of a catalyst particle is formed. It always has an enough concentration of vacant sites available both for transport and adsorption. In the central part, $\Theta$ easily overcomes the percolation threshold. After this, surface transport peculiarities in the central part are similar to those predicted by kinetic modeling (2)-(4) above $\Theta=1 / 2$. The main contribution into the output surface flux are given by not long-path and labored diffusion delivery from the central part to the periphery, but direct adsorption onto vacancyenriched peripheral adsorbing zone that is gradually constricted with increase of $J_{\text {inp }}$. These peculiar properties are illustrated by Fig. 4. If we adopt the spatially averaged coverage $\langle\Theta\rangle$ as a representative parameter of the catalyst surface, we will see that the functions $J_{\text {out }} / J_{\text {inp }}=f(\langle\Theta\rangle)$ and $J_{\text {out }} / J_{\text {inp }}=\varphi\left(J_{\text {inp }}\right)$ have the extremum at $\langle\Theta\rangle=0.25$. It corresponds to beginning of the surface transport slowing down in the central $(\Theta(0)>0.5)$ part of the region and switching over to the direct delivery through peripheral zone. Just due to this mechanism the dependence $\langle\Theta\rangle=F\left(J_{\text {inp }}\right)$ is monotonous and has no peculiarities mentioned above.

Therefore, we may conclude that the crude kinetic model being unable to describe these fine details in surface distribution of atoms predicts well the coverage vs. flux dependence in the central part of catalyst surface, where the surface concentration is more uniform. One may conclude that kinetic modeling together with the MC modeling using a probe particle migration may be rather promising for simulations of the real growth.

\section{Peculiarities of NW doping according to the kinetic model}

Let us add the second (doping) component into the gas phase and assume that respective dopant has nearly the similar parameters as to molecular adsorption, bulk diffusion and the surface transport as a silicon adatom. The kinetic equations for coverage with silicon atoms $(\Theta)$ and doping agent $(\Phi)$ should be rewritten as follows:

$$
\begin{aligned}
& \sigma \frac{d \Theta}{d t}=J_{1}(1-\Theta-\Phi)-\sigma \Theta\left(\beta_{1}+1 /\left\langle t_{1}\right\rangle\right), \\
& \sigma \frac{d \Phi}{d t}=J_{2}(1-\Theta-\Phi)-\sigma \Phi\left(\beta_{2}+1 /\left\langle t_{2}\right\rangle\right)
\end{aligned}
$$

Here, the subscripts " 1 " and " 2 " are related to the matrix and doping species. The initial conditions for (5) are $\Theta(0)=\Theta_{i}$ and $\Phi(0)=\Phi_{i}$. We will be interested in kinetics of NW bulk doping level near the interface during growth, denoting as $C_{I}=C_{I}(t)$, or the impurity distribution along $\mathrm{NW}$ length $C_{I}=C_{I}(z)$. For simplicity, we will consider the case when the fluxes ratio $\xi=J_{2} / J_{1}$ does not exceed the maximum solubility for the given dopant in the silicon matrix. This is the case of $\Phi / \Theta<<1$ and according to MC simulations for similar species we have that $\left\langle t_{1}\right\rangle^{-1}=\alpha D_{01} \cdot(1-\theta-\Phi)^{n} / R^{2}$, $\left\langle t_{2}\right\rangle^{-1}=\alpha D_{02} \cdot(1-\theta-\Phi)^{n} / R^{2}$, and $\left\langle t_{1}\right\rangle \approx\left\langle t_{2}\right\rangle$ as well as $\beta_{1} \approx \beta_{2}$. We studied the system (3) numerically, and results may be summarized as follows.

(i) In any case $C_{I}(t)_{\mid t \rightarrow \infty} \rightarrow \xi$, but the characteristic time $\tau_{I}$ of this transition process depends strongly on the initial coverage with silicon adatoms $\Theta_{i}=\Theta(0)$ and on the surface to bulk transport coefficient ratio $r_{s b}=\frac{D_{0}}{D_{b}} \cdot \frac{\alpha \lambda}{\kappa R}$. For typical nanosized objects $(R \approx 100 \lambda) r_{s b} \approx 0.1 \cdot \frac{D_{0}}{D_{b}}$. Therefore, this effect is the size-sensitive one.

(ii) For $\Theta_{i}=\Phi_{i}=0$, we have $C_{I}(t)=\xi$ just from the beginning of the growth process and uniformly doped NW should grow. However, just this mode is not easily expected, because of preliminary contact of catalyst particles or droplets with the silicon substrate takes place before growth.

(iii) If $\Phi_{i}=0, \Theta_{i} \geq 0.5$ and $D_{0} / D_{b} \geq 10^{3}$, the characteristic time $\tau_{I}$ became comparable with the total time of growth (tens of minutes). As a result, $C_{I}(t)$ slowly increases from 0 up to $\xi$. The bottom and middle part of NW proves to be low-doped and only top part turns out to be doped according to macroscopic partial pressure of a dopant gas in the gas mixture $\xi=J_{2} / J_{1}$. This effect may be used for heterostructure formation during growth.

(iv) Any manipulations with the flux of doping species, for example change of $J_{2}$ in time to achieve the 
desired length distribution of impurity along the NW, are ineffective while $\Theta_{0} \geq 0.5$ due to time lag of doping level response.

(v) The lag of doping level from $\xi=\xi(t)$ practically disappears when $D_{0} / D_{b} \leq 10$ or/and $\Theta_{0} \approx 0.1 \ldots 0.2$. Therefore, the mode of small fluxes both ( $J_{1}$ and $J_{2}$ ) should be used to achieve more or less uniform bulk NW doping along its length.

The MC simulations reveal an additional important feature of doping in the case of high value of $\Theta$. The narrow vacancy-enriched peripheral region mentioned above is the region where $\Theta$ is just about 0.1 during the whole growth process. Due to this effect it is possible to realize selective and uniform doping of NW shell.

\section{The role of slow diffusing components on the surface of catalyst}

The MC experiments with random walk of some probe atom show (Fig. 2b, c) that the presence of practically immobile (slow-diffusing) species suppresses surface transport much more effectively than the presence of the mobile ones does. For immobile species, the coverage of about $0.42 \leq \Theta_{\text {slow }} \leq 0.43$ is enough to completely block most of the surface routs for adatom delivery from the inner part of catalyst surface to the sidewall (Fig. 2d). The respective dependence for $D_{\text {mob }}=D_{0}\left(\Theta_{\text {slow }}\right)$ is shown in Fig. 2e (curve 2). When the fraction of slow-moving adatoms $\Theta_{\text {slow }}$ is smaller than 0.35 , a quasi-linear relation between $\left\langle R^{2}\right\rangle$ and $t$ (or $\langle t\rangle$ and $R^{2}$ at the given $R^{2}$ ) for mobile randomwalking probe atoms proves to be valid (Fig. 2b). Beginning from $\Theta_{\text {slow }} \approx 0.38$ (Fig. 2c), the non-linear relation for $\left\langle R^{2}\right\rangle=4 D_{\text {eff }} t^{\mu}$ takes place, and the so-called "subdiffusion" $(\mu<1)$ is observed [7], while $\mu$ decreases when $\Theta_{\text {slow }} \rightarrow 0.43$. Here, the kinetic modeling similar to (2) and (3) cannot be applied at all. In this critical interval of $\Theta_{\text {slow }}$, slow-diffusing atoms begin to form some coherent groups or aggregates. As a result, a mobile atom is forced to diddle a lot of time to double or skirt them to achieve the boundary of the region. Therefore, $\left\langle R^{2}\right\rangle$ proves to be behind the dynamical time $t$. One can see from Fig. $2 \mathrm{~d}$ that for $0.38 \leq \Theta_{\text {slow }} \leq 0.43$ most of the diffusion paths are temporary blocked and the surface flux toward the periphery of the catalyst dome becomes more and more anisotropic. This effect of anisotropy may be responsible for the appearance of some complex forms (e.g. spring) in the nanostructures.

The consideration of the atom spread kinetics in another MC experiment (Fig. 3) supports the conclusion about the surface transport suppression derived from probe particle random walk. The time of mobile atom residence on the surface at the initial value $\Theta_{\text {mob }}=0.3$ is nearly the same for $\Theta_{\text {slow }} \leq 0.2$ and increases rapidly at threshold value $\Theta_{\text {slow }} \approx 0.4$ with subsequent almost complete blocking at $\Theta_{\text {slow }} \approx 0.6$ and higher, when only peripheral mobile atoms are able to escape from the surface. The role of immobile species seems to be very useful for creating perfect cylinders. Complete suppress of the surface transport leaves the only bulk transport of silicon adatoms to the interface through the catalyst particle. In particular, chlorine-containing complexes used in [12] to suppress nanowire tapering may also act (in addition to the mechanism proposed in [12]) by this mechanism.

Therefore, as to doping of NW we have considered two cases of close and very different local mobilities for matrix $\left(D_{01}\right)$ and impurity atoms $\left(D_{02}\right)$ that demonstrate qualitatively quite different behavior. We also checked the intermediate case (in MC experiment with probe particle) when $D_{01} / D_{02}=1 / 2 \ldots 2$ and $\Theta=\Theta_{1}+\Theta_{2}$. For effective diffusivity, we obtained some weighed mean intermediate value between respective one-component systems. If the share of slowmoving atoms $\left(\Theta_{2}\right)$ at the surface increases, $D_{\text {eff }}$ decreases in comparison with that for a one-component system with the same total coverage.

\section{Conclusion}

Atomic transport on the surface of catalyst was considered by Monte-Carlo simulations. Four different treatments of the experiment were proposed to estimate the effective transport coefficient and to reproduce the real experimental situation. Respective numerical kinetic modeling devoted to the NW growth and doping was added to compare the atomistic and macroscopic approaches. This multilevel modeling proves to be fruitful for better understanding of the obtained results. These results reveal the importance of the role of surface transport and the presence of surface immobile contaminations in the nanowire growth. It was shown that the ratio between the bulk and the surface fluxes through or across the catalyst particle depends critically on the local mobility and the fractions of the different species present on the surface. The presence of a moderate quantity of immobile species (such as byproducts of incomplete silane decomposition) effectively suppresses the flux towards the sidewall.

We observed also the following non-linear effects: (1) complete or partial blockage of surface transport in the presence of slow-moving impurities; (2) appearance of surface flux anisotropy and subdiffusion of mobile atoms when the coverage with slow-moving atoms $\Theta_{\text {slow }} \rightarrow 0.4$; (3) sufficient suppression of the surface transport of equally mobile atoms at high input fluxes and (4) non-proportionality between the input flux of 
impurity and the doping level under the high enough input flux of only one component. The results of simulations allowed to predict some approaches to the shape and doping control during the nanowire growth. In the presence of immobile impurities, the behavior of dopants incorporated into nanowire during growth became more predictable. Both uniform and coaxial doping may be realized independently, due to suppressing or release of surface transport across catalyst particle.

\section{Acknowledgements}

The authors wish to express their sincere gratitude to Dr. T. Kamins (Hewlett Packard) for helpful discussions. The authors also thank Dr. S. Lukyanets (Institute of Physics, National Academy of Sciences of Ukraine) for discussion of target setting for some of prepared MC simulations.

\section{References}

1. A. Efremov, A. Klimovskaya, T. Kamins, B. Shanina, K. Grygoryev, S. Lukyanets // Semiconductor Physics, Quantum Electronics \& Optoelectronics 8 (3), p. 1 (2005).

2. E.I. Givargizov, Growth of filament-like and platelet crystals from vapor. Nauka, Moscow, 1977 (in Russian); D.N. McIlroy, D. Zhang, Y. Kranov and M. Grant Morton // Appl. Phys. Lett. 79, p. 1540 (2001).

3. D.N. McIlroy, A. Alkhateeb, D. Zang // J. Phys.: Condens. Matter 16, R415 (2004).

4. A.I. Klimovskaya, E. G. Gule, I.V. Prokopenko // Proc. of $17^{\text {th }}$ Quantum Electronics Conference (MIEL 2000), Nis, Yugoslavia.

5. A.I. Klimovskaya, I.V. Prokopenko and I.P. Ostrovskii // J. Phys.: Condens. Matter 13, p. 5923 (2001).

6. A.I. Klimovskaya, I.V. Prokopenko, S.V. Svechnikov // J. Phys.: Condens. Matter 14, p. 1735 (2002).

7. L.M. Zelenyy, A.V. Milovanov // Uspekhi fiz. nauk 174, (8), p. 809 (2004).

8. L.K. Orlov, T.N. Smyslova // Semiconductors 40, p. 43 (2006).

9. H. Hould, J. Tobochnik, An introduction to computer simulation methods. Application to physical systems. Part 2, Addison-Wesley Publishing Company, NY, 1988.

10. D.W. Heerman, Computer simulations methods in theoretical physics. Springer Verlag, Berlin, 1986.

11. A. Frank-Kamenetskii, Diffusion and heat transfer in chemical kinetics. Nauka, Moscow, 1987 (in Russian).

12. S. Sharma, T.I. Kamins, R. Stanley Williams // J. Cryst. Growth 267, p. 613 (2004). 